\title{
Contralateral Unidirectional Cavopulmonary Shunt for Single Ventricle Palliation
}

\author{
Anisha Agrawal ${ }^{1}$, Jeevanandam N², Shekhar Saxena ${ }^{2}$, and Roy Varghese $^{2}$ \\ ${ }^{1}$ Topiwala National Medical College \\ ${ }^{2}$ Madras Medical Mission Institute of Cardio Vascular Diseases
}

July 3, 2021

\begin{abstract}
Unidirectional cavo pulmonary shunt supplemented with systemic to pulmonary arterial shunt is often necessary for palliation of single ventricle with unilateral hypoplasia of a pulmonary artery. In rare instances, the adequately sized pulmonary artery is on the contralateral side as the superior caval vein making this anastomosis challenging. This report describes the operative technique involved in construction of the right superior caval vein to left pulmonary artery anastomosis.
\end{abstract}

\section{Title:}

Contralateral unidirectional cavopulmonary shunt for single ventricle palliation

\section{Running Head:}

CONTRALATERAL "SUPER GLENN"

\section{Authors:}

Anisha Agrawal ${ }^{1}$

Jeevanandam, $\mathrm{MD}^{2}$

Shekhar Suman Saxena, MD ${ }^{3}$

Roy Varghese, $\mathrm{MCh}^{4}$

\section{Institutions and Affiliations:}

T. N. Medical College \& BYL Nair Ch. Hospital, Mumbai, India

MBBS Student ${ }^{1}$

Institute of Cardiovascular Diseases, Madras Medical Mission, Chennai, India

Associate Consultant, Cardiac Anesthesiology ${ }^{2}$

Registrar, Cardiac Anesthesiology ${ }^{3}$

Senior Consultant, Pediatric cardiac surgery ${ }^{4}$

\section{Corresponding Author:}

Roy Varghese

Senior Consultant, Pediatric Cardiac Surgery 
Institute of Cardiovascular Diseases, Madras Medical Mission, Chennai, 600037, India

Tel: +91 9840550256, Fax: +91 44 26565859, email: jonroy65@gmail.com

Data availability statement: The authors confirm that the data pertaining to this surgical technique are available within the article and its supplementary materials.

\section{Acknowledgments}

None. No funding to declare.

\section{Disclosures}

Authors declare that they have no conflict of interest.

\section{Institutional Review Board approval}

All procedures followed were in accordance with ethical standards as per institutional and national guidelines.

Word Count: 1446

\section{Abstract:}

Unidirectional cavo pulmonary shunt supplemented with systemic to pulmonary arterial shunt is often necessary for palliation of single ventricle with unilateral hypoplasia of a pulmonary artery. In rare instances, the adequately sized pulmonary artery is on the contralateral side as the superior caval vein making this anastomosis challenging. This report describes the operative technique involved in construction of the right superior caval vein to left pulmonary artery anastomosis.

\section{Introduction}

In patients with single ventricle and unilateral hypoplasia of a pulmonary artery, unidirectional cavo pulmonary shunt (UCPS) performed on the side with the adequately sized pulmonary artery combined with systemic to pulmonary artery shunt on the side with the hypoplastic pulmonary artery ("Super Glenn") provides initial palliation. The UCPS is, in most instances, performed on the ipsilateral side as the superior caval vein (on which side the pulmonary artery is of adequate size) and the systemic to pulmonary artery shunt to the contralateral (hypoplastic) pulmonary artery. Occasionally, however, the adequately sized pulmonary artery and the superior caval vein are on opposite sides making this anastomosis challenging.

This manuscript describes the surgical technique involved in the performance of UCPS between the right superior caval vein and left pulmonary artery at the hilum of the lung across the midline, combined with a systemic to pulmonary artery shunt to the hypoplastic pulmonary artery.

\section{Technique}

A two-year-old female child with diagnosis of tricuspid atresia and pulmonary atresia presented with resting oxygen saturations $\left(\mathrm{SpO}_{2}\right)$ of $70 \%$. She had a duct dependent pulmonary circulation with adequately sized left pulmonary artery (LPA) at the hilum $(\sim 12 \mathrm{~mm})$ and hypoplastic right pulmonary artery (RPA) $\left(^{\sim}\right.$ $3 \mathrm{~mm})$. There was significant long segment narrowing at the pulmonary artery confluence. Ductal stenting performed at the time resulted in "jailing" of the RPA with the stent lying within the LPA in its entirety. Follow up echocardiography showed a severely narrowed LPA regaining adequate size at the hilum.

She now presented at five years of age with significant cyanosis $\left(\mathrm{SpO}_{2} 65 \%\right)$. Evaluation confirmed hypoplasia of the RPA. The LPA, while adequately sized at the hilum, remained narrow at the central portion. The decision taken at the team meeting concluded that a "Super Glenn" offered the best chance of recruiting the hypoplastic RPA for a future Fontan completion. The surgical team was however concerned about the possibility of the single right superior caval vein (SVC) reaching across the midline to the hilum of the left lung. 
The heart and great vessels were approached through median sternotomy. Presence of a single right SVC was confirmed. The diameters of the RPA and the LPA (at the hilum) were approximately $3 \mathrm{~mm}$ and 12 $\mathrm{mm}$ respectively. Following subtotal thymic excision, the innominate vein, SVC, and proximal portions of subclavian and internal jugular veins were extensively mobilised. Division of the azygous vein and right internal mammary vein facilitated this mobilization. The RPA and LPA were dissected from surrounding structures until the hilar regions on either side. The ductal stent was dissected and looped.

Cardiopulmonary bypass was initiated with aortic cannulation, followed by cannulation of the innominate vein and inferior caval vein. The perfusate was maintained at normothermia and the operation was performed with the heart beating. The ductal stent was then interrupted using a vascular clip. The stump of the main pulmonary artery was divided to aid in the mobilisation of the LPA. The SVC was transected at the cavo atrial junction and its cardiac end oversewn. The ductal stent was found extending till the hilum on the left side, stopping short immediately proximal to the upper lobe branch. The LPA was transected at the termination of the stent and further mobilised into the hilar region taking care to protect the phrenic nerve. The transected SVC was then brought across the midline posterior to the ascending aorta, and anastomosed to the transected LPA in an end-to-end manner. Thus, a contralateral cavo pulmonary shunt between the right SVC and the LPA was achieved

To promote growth of the hypoplastic RPA, a $5 \mathrm{~mm}$ expanded poly tetra fluoro ethylene (ePTFE) vascular graft was interposed between it and the ascending aorta. The graft was anastomosed to the RPA and aorta in an end-to-side and side-to-side manner, respectively (Figures 1, 2, \& 3 ).

The patient was weaned off cardiopulmonary bypass after sufficient rewarming. On measurement, the Glenn pressures were found to be elevated $(22 \mathrm{mmHg})$. The RPA origin from the arterial duct was found compressing the SVC beneath the aorta much like a vascular sling. Division of the duct resulted in abolishment of the gradient. The oxygen saturations rose to $92 \%$.

Echocardiography demonstrated laminar flows in the UCPS and adequate flows in the aorto-pulmonary shunt.

The patient was discharged on the $8^{\text {th }}$ day, with oxygen saturations of $95 \%$. We anticipate possible completion of single ventricle palliation once the hypoplastic RPA grows to an appropriate size.

\section{Comment}

Single ventricle with unilateral hypoplasia of a pulmonary artery precludes performance of bidirectional cavo pulmonary shunt (BCPS) due to insufficient flows into the hypoplastic pulmonary vascular bed. Fontan completion would, likewise, fail in these circumstances for similar reasons ${ }^{1}$. In these situations, Tachi et al and Casella et al advocated targeted increase of pulmonary blood flows to the hypoplastic lung while protecting the lung bearing the cavo pulmonary shunt from the elevated systemic arterial pressures ${ }^{2,3}$.

We divided the ductal origin of the pulmonary artery to which the aorto pulmonary shunt was inserted since this was forming a sling around the superior caval vein as it traversed the midline to the opposite side. This maneuver, in addition, protects the cavo pulmonary shunt.

In most patients undergoing the "Super Glenn", the cavo pulmonary shunt can be performed to the ipsilateral pulmonary artery which is adequately sized. The systemic to pulmonary arterial shunt is then performed to the hypoplastic pulmonary artery which in most instances lies on the contralateral side. The patient in this report had the SVC and pulmonary artery on opposite sides making the anastomosis more challenging. With adequate mobilization of all structures involved, this could be completed with relative ease.

Adequately sized pulmonary arteries (among other criteria) are a prerequisite for successful performance of the Fontan operation ${ }^{1,4}$. It is hoped that on follow up, the RPA would show adequate growth and arborization to permit completion of the final stage of the single ventricle palliation in this patient. At the time of completion, we would anastomose the RPA to the transversely lying SVC to which we would connect 
the inferior caval vein. If in the not so ideal situation of the RPA failing to show adequate growth, this would be final palliation for the child who would then have a better quality of life and oxygen saturations.

In conclusion, we have described an easily reproducible technique for the performance of a unidirectional cavo pulmonary anastomosis to the pulmonary artery that is contralateral to the superior caval vein in patients with single ventricle and unilateral hypoplasia of a pulmonary artery.

\section{References :}

1. Stern HJ. Fontan "Ten Commandments" revisited and revised. Pediatr Cardiol. 2010;31(8):1131-4.

2. Tachi M, Murata M, Ide Y, et al. Efficacy of the 'intrapulmonary-artery septation' surgical approach for Fontan candidates with unilateral pulmonary arterial hypoplasiat. Eur $J$ Cardiothorac Surg. 2016;49(1):183-7.

3. Casella SL, Kaza A, Del Nido P, Lock JE, Marshall AC. Targeted Increase in Pulmonary Blood Flow in a Bidirectional Glenn Circulation. Semin Thorac Cardiovasc Surg. 2018;30(2):182-8.

4. Fontan F, Baudet E. Surgical repair of tricuspid atresia. Thorax . 1971;26(3):240-8

\section{Figure legends:}

Figure 1: Operative view of the cavo pulmonary shunt

Figure 2: Operative view of the aorto pulmonary shunt

Figure 3: Sketch showing end-to-end anastomosis of SVC to LPA and aorto pulmonary shunt to RPA.

Abbreviations: Dia: Diameter. IVC: Inferior caval vein. LPA: Left pulmonary artery. PDA: Persistent ductus arteriosus. RPA: Right pulmonary artery. SVC: Superior caval vein.

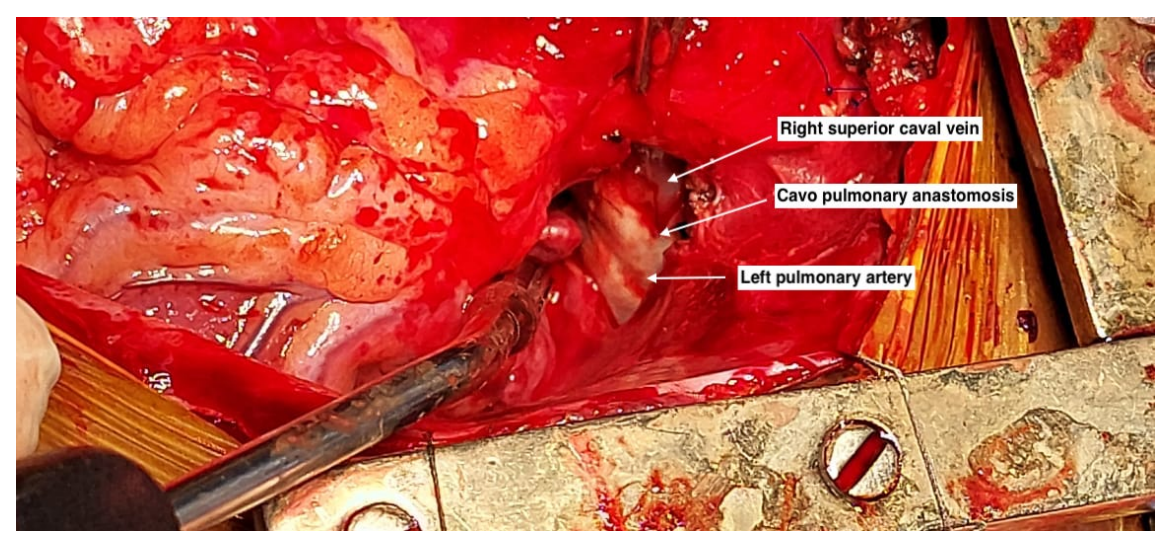



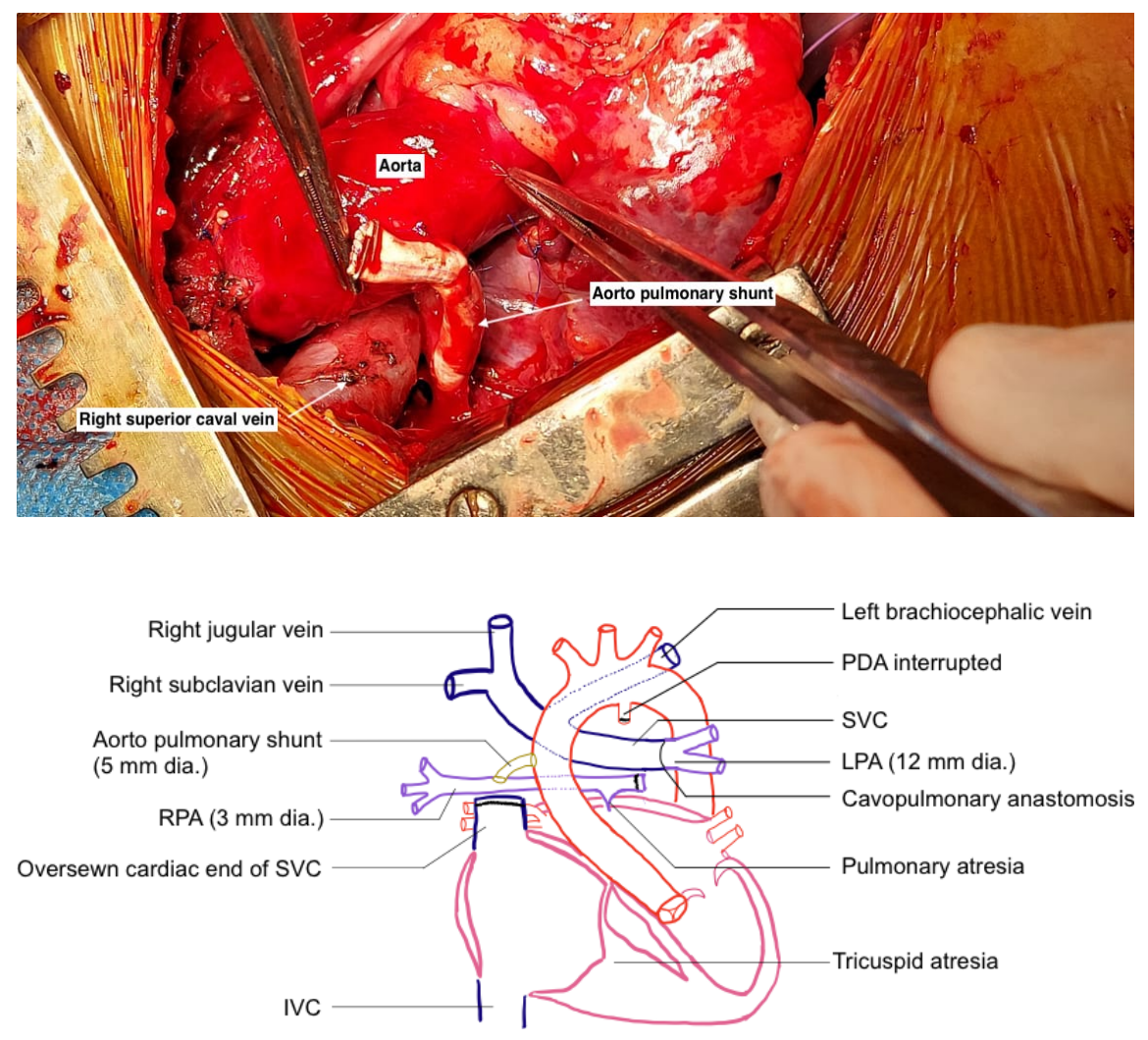